\title{
Razonamiento y racionalidad desde la psicología evolucionista*
}

\author{
Reasoning and Rationality from the Viewpoint \\ of Evolutionary Psychology
}

Jonatan García Campos

\begin{abstract}
Resumen
La psicología evolucionista considera que la mente humana está constituida por módulos producto de la selección natural. Se ha argumentado a favor de varios módulos, uno de ellos diseñado para lidiar con la detección de tramposos en contratos sociales. Dado este y otros módulos, los psicólogos evolucionistas sostienen que están infundadas nuestras preocupaciones en torno a la "interpretación pesimista de la racionalidad humana". Los propósitos de este trabajo son i) explorar cuáles pueden ser los resultados en relación con la racionalidad, si se aceptan las tesis defendidas por los psicólogos evolucionistas, y ii) evaluar si dichos resultados pueden ser usados para rechazar la interpretación pesimista de la racionalidad. Sostendré que la psicología evolucionista puede ser usada solo para rechazar una versión de la interpretación pesimista de la racionalidad, pero que existe otra versión de dicha interpretación que es compatible con esa postura psicológica e incluso puede ser explicada por ella.
\end{abstract}

Palabras clave: racionalidad - razonamiento - psicología evolucionista - modularidad

\begin{abstract}
Evolutionary psychology claims that the human mind is formed by modules which are product of natural selection. Different modules have been postulated, one of them is thought to have been designed to detect cheaters in social intercourses. With this module and some others, evolutionary psychologists consider that we do not have to worry about the pessimistic interpretation of human rationality. The purposes of this article are: i) to explore the results in relation to rationality, if we would accept the theses defended by evolutionary psychologists, and ii) to evaluate if those results can be used to reject the pessimistic interpretation of rationality. I shall argue that evolutionary psychology can be used to refuse just one version of the pessimistic interpretation of rationality, but there is another version of that interpretation that can be compatible with and even explained by this psychological approach.
\end{abstract}

Keywords: rationality - reasoning - evolutionary psychology - modularity

* 12 Diciembre 2010. Aceptado en versión revisada: 25 Octubre 2011.

† Instituto de Ciencias Sociales de la Universidad Juárez del Estado de Durango. Para contactar al autor, por favor escriba a: jongarcam@yahoo.com.mx.

* Este trabajo ha sido financiado por el Programa de Mejoramiento del Profesorado, Proyecto Justificación y Racionalidad (PROMEP/103.5/11/6773).

Metatheoria 2(1) (2011): 79-100. ISSN 1853-2322.

(C) Editorial de la Universidad Nacional de Tres de Febrero. Publicado en la República Argentina. 


\section{Razonamiento y racionalidad desde la psicología evolucionista}

La psicología evolucionista considera que la mente humana está constituida por módulos producto de la selección natural. Se ha argumentado a favor de varios módulos, uno de ellos diseñado para lidiar con la detección de tramposos en contratos sociales. Dado este y otros módulos, los psicólogos evolucionistas sostienen que están infundadas nuestras preocupaciones en torno a la "interpretación pesimista de la racionalidad humana". Los propósitos de este trabajo son explorar cuáles pueden ser los resultados en relación con la racionalidad, si se aceptan las tesis defendidas por los psicólogos evolucionistas, y evaluar si dichos resultados pueden ser usados para rechazar la interpretación pesimista de la racionalidad.

Dado lo anterior, en este trabajo no examinaré la legitimidad de los principios teóricos defendidos por la psicología evolucionista, ni la validez de los experimentos particulares desarrollados por los seguidores de este tipo de psicología, sino que me centraré en clarificar y analizar las implicaciones que dichos principios y experimentos tienen para el debate en torno a la racionalidad. En este campo particular, defenderé que la psicología evolucionista puede ser usada solo para rechazar una versión de la interpretación pesimista de la racionalidad, pero que existe otra versión de dicha interpretación que es compatible con esa postura psicológica e incluso puede ser explicada por ella. Además, propondré que los psicólogos evolucionistas están implícitamente aceptando un criterio particular para entender los estándares normativos con los que se debe evaluar el razonamiento.

La estructura del trabajo es la siguiente. En primer lugar, reviso cierta evidencia sobre el razonamiento, que ha sido usada para defender la interpretación pesimista de la racionalidad. Posteriormente, expongo las tesis centrales de la psicología evolucionista así como su estrategia metodológica, para enseguida examinar lo que se ha denominado el módulo de detección de tramposos. En tercer lugar, exploro las implicaciones en el debate acerca de la racionalidad que tienen las tesis defendidas por los psicólogos evolucionistas y evalúo si dichas implicaciones pueden ser usadas para rechazar la interpretación pesimista de la racionalidad. Por último, ofrezco razones que sugieren que los psicólogos evolucionistas están proponiendo, quizá sin ser completamente conscientes de ello, una manera distinta de entender la racionalidad humana.

\section{La evidencia en contra de la racionalidad humana}

Algunos filósofos consideran que se pueden distinguir tres maneras de estudiar la razón, a saber, los estudios de tipo descriptivo, normativo y evaluativo (Samuels et al. 2004). El primer tipo de estudios consiste en describir cómo es que el ser humano de hecho razona, así como descubrir los mecanismos y procesos psicológicos que subyacen en los patrones de razonamiento observados. Aquí se ubican las distintas teorías del razonamiento defendidas por los psicó- 
logos cognitivos, entre ellas, la psicología evolucionista que abordaré más adelante. El estudio de tipo normativo, a diferencia del estudio de tipo descriptivo, no da cuenta de cómo los seres humanos razonan, sino de cómo deben razonar. Una de las metas centrales en este tipo de estudios es indagar cuáles son las reglas o principios que indiquen qué es razonar correctamente. Por su parte, el estudio de tipo evaluativo busca determinar el valor que tiene el razonamiento de acuerdo con un estándar normativo particular. Dados ciertos criterios normativos, quienes realizan un estudio de tipo evaluativo determinan cuándo el razonamiento cumple con tales criterios.

Cada uno de los resultados o descubrimientos provenientes de estos tres distintos tipos de estudio de la razón se pueden relacionar entre sí. Por ejemplo, cuando se evalúan los procedimientos usados por un individuo se parte ya de un conjunto de normas que se contrasta con una descripción de lo que el individuo realmente hace, cognitivamente hablando. Del mismo modo, se ha argumentado que la manera en que el ser humano procede en el ámbito cognitivo tiene repercusiones en el modo en que se pueden evaluar sus procedimientos cognitivos, ${ }^{1}$ e incluso, aunque ampliamente debatido, se ha sostenido que las normas pueden adquirir validez o invalidez a partir del modo en que queda descrito el proceder cognitivo de los sujetos. ${ }^{2}$ Muchas veces, como se verá a lo largo de este trabajo, los distintos tipos de estudios de la razón están tan íntimamente ligados que resulta difícil trazar líneas claras entre estos. Sin embargo, conservaré esta distinción como una herramienta de análisis que servirá para entender con mayor claridad algunas de las posiciones que se presentarán posteriormente.

Uno de los debates en donde confluyen los tres tipos de estudios de la razón arriba descritos surge de una serie de resultados provenientes de la psicología cognitiva del razonamiento, en particular de un programa conocido como la "tradición de heurística y sesgo" liderado por Daniel Kahneman y Amos Tversky (Kahneman, Slovic \& Tversky 1982, Gilovich, Griffin \& Kahneman 2002). Algunos filósofos consideran que el debate actual en torno a la racionalidad humana no puede ignorar algunos de esos trabajos (Goldman 1993), puesto que tales trabajos, han creído muchos, apoyan una visión pesimista de la racionalidad humana. Dichos trabajos se centran tanto en la toma de decisiones y razo-

\footnotetext{
${ }^{1}$ Hay un conjunto de trabajos en filosofía y ciencias cognitivas, dentro del que destaca Cherniak (1986), que ha planteado que la noción de racionalidad debe ser replanteada o acotada a partir de modelos (psicológica y computacionalmente) realistas. Uno de los puntos discutido dentro de estos trabajos, por ejemplo, es que la consistencia lógica es un ideal epistémico que no podemos satisfacer dado que sobrepasa las capacidades computacionales de los seres humanos (véase también Goldman 1993).

${ }^{2}$ La idea de que los estándares normativos tienen su origen en el modo en que los seres humanos de hecho razonan puede ser entendido como un acercamiento psicologista a la racionalidad (Elio 2002). Dos acercamientos a la racionalidad pueden ser contrastados con el psicologismo. Uno de ellos sería defender un acercamiento en el que las normas de racionalidad se encuentran en un reino de verdades fuera del ámbito humano. Otro acercamiento a la racionalidad sería aquel que sostuviese que los estándares normativos pueden ser entendidos como invenciones que pueden ser usadas para producir propósitos particulares. Mientras que se podría considerar el primero de estos acercamientos como un platonismo, al segundo acercamiento se le podría denominar constructivismo (Elio 2002).
} 
namiento inductivo (Kahneman, Slovic \& Tversky 1982), como en el razonamiento deductivo (Evans 1991).

Uno de los estudios de razonamiento deductivo más importantes es lo que se conoce como "tarea de selección de tarjetas" llevado a cabo por primera vez por Peter Wason (1966). En un caso típico de tarea de selección se les presenta a los sujetos cuatro tarjetas como aparecen a continuación y posteriormente se les explica lo que deben realizar.
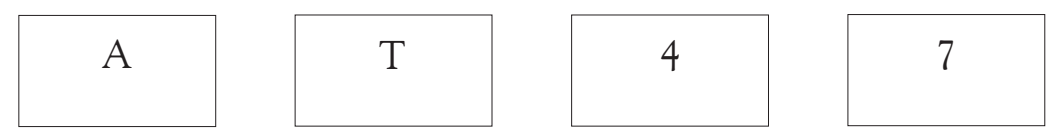

Todas las cartas anteriores tienen de un lado un número y del otro una letra del abecedario. Tu trabajo es determinar cuáles de estas cartas tienes que necesariamente voltear para probar la verdad de la oración: "si hay una vocal en un lado de la carta, entonces hay un número par en su otro lado".

Wason y otros psicólogos descubrieron que comúnmente los sujetos no responden correctamente a este tipo de tareas, pues, aun cuando la respuesta correcta son las tarjetas A y 7, la mayoría de sujetos (alrededor del 75\%) solo consideran que se debe voltear la tarjeta A, o las tarjetas A y 4 (Evans \& Over 1996).

Dado que se hicieron un número importante de experimentos para estudiar el razonamiento deductivo (e inductivo) y de ellos se obtuvo evidencia que parecía señalar errores sistemáticos de razonamiento, se sostuvo que los procesos cognitivos usados por los seres humanos se desvían de las normas de racionalidad apropiadas. ¿Cuáles son estas normas? Algunos psicólogos cognitivos no suelen ser explícitos en relación con los criterios o estándares que usan para evaluar el razonamiento pero la mayoría de ellos acepta lo que Edward Stein (1996) denomina la visión estándar de la racionalidad (VER): "de acuerdo con esta visión ser racional es razonar en concordancia con los principios de razonamiento que están fundados en las reglas de la lógica, la teoría de la probabilidad u otras teorías formales" (p. 4).

Es preciso indicar que si bien la definición de la VER puede parecer vaga, generalmente los psicólogos que asumen dicha visión consideran que, en relación con los estudios de razonamiento deductivo, son los principios de la lógica clásica los que deben ser usados para derivar las normas de razonamiento correcto. Por ejemplo, cuando algunos psicólogos consideran que en el caso de la tarea de selección de tarjetas los sujetos cometen un error de razonamiento al seleccionar únicamente la tarjeta "A", lo hacen porque asumen que la tabla de verdad del condicional establece que la única posibilidad de que la tabla de verdad sea falsa es cuando el antecedente es verdadero y el consecuente es falso. Así, si lo que se desea probar es "si hay una vocal en un lado de la carta, entonces hay un número par en su otro lado" solo voltear la tarjeta "A", por ejemplo, no probaría la verdad del condicional. En otras palabras, lo que hace que los psicólogos de la tradición de heurística y sesgo consideren ciertas respuestas como incorrectas se debe a que presuponen un estándar normativo particular, a saber, la VER. 
En tanto que muchos psicólogos cognitivos del razonamiento asumieron la VER y los experimentos parecen mostrar que los individuos no satisfacen los estándares de dicha visión, se defendió la idea de que los seres humanos comenten sistemáticamente errores de razonamiento (Piatelli-Palmarini 1994, 2005). A esta interpretación de los estudios se la conoce como "la interpretación pesimista de la racionalidad humana”. A mi juicio, hay por lo menos dos modos de entender esta interpretación:

(I) Muchos fenómenos donde los juicios de razonamiento se desvían de las normas de racionalidad se explican por el hecho de que el razonamiento humano puede fundarse en mecanismos que comúnmente no satisfacen las normas de la VER.

(II) Muchos fenómenos donde los juicios de razonamiento se desvían de las normas de racionalidad se explican por el hecho de que los únicos mecanismos de razonamiento que los seres humanos poseen no satisfacen las normas de la VER.

Como es fácil notar, la versión pesimista (II) es más radical que la versión pesimista (I) ${ }^{3}$ sin embargo, es posible encontrar las dos versiones en diversos psicólogos cognitivos del razonamiento. ${ }^{4}$

A diferencia de la versión (I), la versión (II) está dando por hecho que todos los mecanismos de razonamiento del ser humano dependen siempre de heurísticas normativamente inapropiadas. Mientras que la versión pesimista (I) considera que, si bien los mecanismos de razonamiento pueden seguir heurísticas que suelen ser problemáticas, es probable que la mente humana también contenga mecanismos (o que mediante el aprendizaje pueda adquirir estrategias que den lugar a procesos) que sigan las reglas de la lógica y la matemática. Más adelante volveré a esta distinción; de momento, deseo señalar que la interpretación pesimista de la racionalidad es el resultado de una evaluación del razonamiento que depende de un estándar normativo particular, i.e., la VER.

\section{La psicología evolucionista}

En los últimos años un grupo de psicólogos evolucionistas, entre ellos Leda Cosmides y John Tooby, han criticado fuertemente la interpretación pesimista de la racionalidad humana. Los psicólogos evolucionistas comparten i) una descripción de la estructura de la mente que se conoce como la teoría de la modularidad masiva; y ii) la estrategia metodológica que deben seguir, especialmente afirman que consideraciones evolutivas deben jugar un papel central en la formación de hipótesis psicológicas. Grosso modo los psicólogos evolucionistas definen de este modo su proyecto teórico:

\footnotetext{
${ }^{3}$ La expresión "muchos de los fenómenos" al inicio de las dos versiones de la interpretación pesimista se debe a que podría haber fenómenos que se desvían de las normas de racionalidad pero que se explican por algo distinto a los mecanismos de razonamiento. Por ejemplo, los sujetos pueden ejecutar inadecuadamente una tarea cognitiva debido a la falta de atención puesta en la tarea, el cansancio, problemas de memoria, etc.

${ }^{4}$ Piatelli-Palmarini (2005) puede representar la versión pesimista (II), mientras que el trabajo del propio Kahneman y su grupo puede ser interpretado como defendiendo la versión pesimista (I).
} 
El objetivo de investigación en la psicología evolucionista es descubrir y entender el diseño de la mente humana. La psicología evolucionista es un acercamiento a la psicología, en el que el conocimiento y los principios de la biología evolucionista son usados para investigar la estructura de la mente... Bajo esta visión, la mente es un conjunto de máquinas procesadoras de información que fueron diseñadas por la selección natural para resolver problemas adaptativos a los que nuestros ancestros cazadores-recolectores se enfrentaron (Cosmides \& Tooby 1997).

\section{a) Tesis centrales y estrategia metodológica de la psicología evolucionista}

Los psicólogos evolucionistas aceptan lo que se denomina la hipótesis de la modularidad masiva. A grandes rasgos, la hipótesis de la modularidad masiva es la afirmación de que la mente humana está compuesta en su mayoría o completamente por mecanismos cognitivos altamente especializados o módulos. ${ }^{5}$ Entre los rasgos que caracterizan a los módulos están:

- Los módulos son mecanismos computacionales innatos. A diferencia de las posturas empiristas, la psicología evolucionista considera que gran parte de la estructura de la mente es innata. Los psicólogos evolucionistas rechazan lo que ellos denominan el "modelo estándar de ciencia social" en el que el mundo social organiza e inyecta significado a las mentes individuales que originalmente están "vacías” (Cosmides \& Tooby 1992, 1997).

- Los módulos son producto de la selección natural. Los psicólogos evolucionistas afirman que la estructura cognitiva de los seres vivos es producto de la selección natural. Bajo esta perspectiva, las mentes están compuestas por adaptaciones que tuvieron su origen durante la historia evolutiva de las especies. Las mentes, según la psicología evolucionista, fueron "diseñadas" por la selección natural para resolver problemas adaptativos, esto es, "problemas evolutivos recurrentes cuya solución promovía la reproducción” (Cosmides \& Tooby 1994, p. 87).

- Los módulos son mecanismos de dominio específico. Los psicólogos evolucionistas conciben los módulos como mecanismos computacionales que son "específicos de dominio" en oposición a dispositivos de "dominio general". Decir que una estructura es específica de dominio significa que está dedicada a solucionar una clase de problemas en un dominio restringido. ${ }^{6}$

\footnotetext{
${ }^{5}$ Los psicólogos evolucionistas consideran que llegan a esa visión de la arquitectura de la mente a partir de varios principios, entre ellos: el cerebro es un sistema físico que funciona como una computadora; los circuitos neuronales de los humanos son producto de la selección natural para resolver problemas que sus ancestros enfrentaron durante la historia evolutiva de la especie, la razón por la cual tenemos un conjunto de circuitos neuronales en lugar de otro es que el circuito que tenemos fue mejor para resolver los problemas a los que nuestros ancestros se enfrentaron durante la historia evolutiva de nuestra especie; diferentes circuitos neuronales están especializados para resolver diferentes problemas adaptativos; y, para decirlo metafóricamente, "los cráneos modernos albergan una mente de la Edad de Piedra" (Cosmides \& Tooby 1997).

${ }^{6}$ Una de las razones que presentan Cosmides \& Tooby (1997) para sostener que la mente debe contener procesos especializados es que en la naturaleza raramente un órgano puede servir para solucionar igualmente bien un mismo problema (un argumento similar aparece en Carruthers 2006).
} 
De este modo, para la psicología evolucionista la mente humana está en su mayoría o quizás enteramente compuesta por módulos innatos, entendidos como mecanismos computacionales, específicos de dominio, producto de la selección natural. Otras características de estos módulos es que se desarrollan en todos los seres humanos normales, su desarrollo no requiere de un esfuerzo consciente o de instrucción formal (Cosmides \& Tooby 1992, 1997). Esta noción de modularidad se conoce como la noción darwiniana de módulo. ${ }^{7}$

Una meta central de la psicología evolucionista es construir y corroborar hipótesis acerca de la visión de modularidad que ellos consideran constituye la estructura de la mente humana. A mi juicio, la estrategia metodológica de la psicología evolucionista puede dividirse en tres etapas. ${ }^{8}$ La primera etapa tiene como meta generar hipótesis verosímiles acerca de los módulos darwinianos. Esta etapa parte de dos ideas: a) el ambiente en el que los humanos evolucionaron fue muy diferente al ambiente actual y b) los ancestros humanos gastaron más del 99\% de la historia evolutiva en sociedades cazadoras-recolectoras. ${ }^{9}$ Estas dos ideas son la base en la que los psicólogos evolucionistas se fundan para tratar de determinar, en la medida de lo posible, los problemas de procesamiento de información que los ancestros humanos tuvieron que enfrentar en lo que se ha llamado "ambiente de adaptación evolutiva" (AAE). El punto más importante consiste en aquellos problemas de adaptación cuya solución exitosa pudo haber contribuido de manera directa o indirecta a un éxito reproductivo.

Una vez que un problema adaptativo ha sido caracterizado, en la segunda etapa, los teóricos formulan hipótesis acerca de un módulo que pudo haber hecho un buen trabajo en la solución de un problema en el AAE. Una vez que se han sugerido hipótesis verosímiles, la tercera y última etapa de investigación en la psicología evolucionista es, por un lado, corroborar las hipótesis indagando si los seres humanos contemporáneos de hecho tienen los módulos que ellos postulan y, por otro lado, descartar hipótesis que puedan explicar de otra manera los resultados empíricos. La evidencia generalmente proviene de estudios

\footnotetext{
${ }^{7}$ Es necesario indicar que la tesis de la modularidad masiva defendida por los psicólogos evolucionistas resulta más radical que la primera descripción modular de la mente, defendida por Jerry Fodor (1983). De acuerdo con este filósofo, la estructura modular de la mente está restringida a los "sistemas de insumo" (input systems), que son responsables del procesamiento del lenguaje y la percepción. Las características de los módulos son, según Fodor, mecanismos específicos de dominio, cada módulo cuenta con su transductor, sus outputs o resultados son no conceptuales, los módulos son innatos, asociados a una estructura específica del cerebro, mandatorios, rápidos en sus procesamientos, informacionalmente encapsulados e inaccesibles. Si bien los psicólogos evolucionistas aceptan la tesis fodoriana de que los sistemas de insumos son modulares, también aceptan que las denominadas capacidades centrales, tales como el razonamiento, pueden tener una estructura modular.

${ }^{8}$ Estas tres etapas siguen la idea de Cosmides \& Tooby (1997) de que la estrategia de la psicología evolucionista es similar al trabajo de ingeniería, en el que primero se ubican los problemas a solucionar, después se planean posibles propuestas y, por último, se prueba si dichas propuestas pueden solucionar los problemas.

${ }^{9}$ Cosmides \& Tooby afirman "nuestros ancestros vivieron en grupos pequeños y nómadas de pocas docenas de individuos que obtenían su alimento diario recolectando plantas y cazando animales. De hecho, cada uno de nuestros ancestros vivió en un "viaje de campamento" que duraba una vida entera, y este estilo de vida subsistió por más de 10 millones de años [...]. La agricultura apareció en la Tierra solo hace 10.000 años, y no fue hasta hace 5.000 años que la mitad de la población humana estuvo ocupada en la agricultura en lugar de la caza y la recolección” (1997, Principio 5).
} 
de laboratorio, similares a los que llevan a cabo los psicólogos de la tradición de heurística y sesgo. Cuando diversos trabajos experimentales apuntan a una misma dirección, según los psicólogos evolucionistas, se puede sostener con cierto fundamento la existencia de un módulo.

\section{b) El módulo de detección de tramposos}

Según los psicólogos evolucionistas, en el AAE de los ancestros homínidos eran comunes los casos en los que los individuos se encontraban en situaciones de intercambio en las que el beneficio que alguien recibía de otro individuo podía ser significativamente mayor que el costo del donante. En una sociedad de cazadores y recolectores había ocasiones en que un cazador podía tener un día afortunado y tuviera comida en abundancia, mientras que ese mismo día otro cazador no tuviera suerte y estuviera cerca de morir de hambre. Si el cazador exitoso diera algo de comida al cazador que no ha tenido éxito en lugar de atiborrarse de comida, esto podría tener un mínimo efecto negativo para el donante puesto que los nutrientes que pudo haber recibido por el exceso de comida podrían ser de utilidad en el futuro; mientras que para el que recibe la comida el beneficio podría ser mucho mayor, pues quizá, de no recibirla, podría morir de hambre. En muchas ocasiones, las personas ayudan a individuos con los que no tienen parentesco, e incluso existe evidencia que sugiere que los primates no humanos hacen frecuentemente lo mismo. Este tipo de altruismo presenta una encrucijada evolucionista, debido a que si el rasgo que hace a un organismo menos proclive a ayudar a otros individuos aparece en la población, entonces dicho organismo será mucho más apto, y así este rasgo se propagaría gradualmente a toda la población.

Una solución a este problema fue propuesta por Robert Trivers (1971) quien notó que, si bien el altruismo es una mala idea desde un punto de vista evolucionista, el altruismo recíproco tiene resultados muy distintos. ${ }^{10} \mathrm{Si}$ un par de cazadores puede contar cada uno con la ayuda del otro cuando uno tiene abundancia de comida y el otro no ha tenido éxito, entonces dichos cazadores se encontrarán a la larga en una mejor situación que aquellos cazadores que no presenten este comportamiento. Aquellos organismos con los genes o conjunto de genes que los inclinen a tener intercambios recíprocos o intercambios sociales serán con el paso del tiempo más aptos que los organismos de su misma especie que no tengan estos genes. Pero, obviamente, los intercambios sociales son vulnerables al engaño. Debido a que el ser humano es capaz de tener relaciones estables de intercambio social, Cosmides \& Tooby (1992) conjeturan que los seres humanos deben contar con uno o más módulos darwinianos, cuyo trabajo es reconocer los acuerdos de intercambios recíprocos y detectar a los tramposos que aceptan los beneficios de tales acuerdos pero que no están dispuestos a pagar el costo que estos conllevan. Los psicólogos evolucionistas afirman:

${ }^{10}$ El trabajo de Robert Axelrod (1996) también es seguido por los psicólogos evolucionistas. 
el intercambio social no pudo evolucionar en una especie a menos que aquellos que están involucrados en dicho intercambio sean capaces de detectar tramposos, esto es, individuos que toman los beneficios de otros sin proveer a los otros de una retribución (Ermer, Cosmides \& Tooby 2007, p. 138).

De este modo, Cosmides \& Tooby (1992) ${ }^{11}$ presentan como hipótesis la existencia de una facultad de cognición social, en la que se encuentra uno o más módulos especializados en la detección de tramposos. ${ }^{12} \mathrm{~A}$ esta hipótesis se la denomina como la hipótesis de la detección del tramposo. ${ }^{13}$

Siguiendo el programa de investigación de la psicología evolucionista, si la hipótesis de la detección del tramposo es correcta, entonces debe encontrarse evidencia en los seres humanos contemporáneos que señale la existencia de un módulo para detectar tramposos, y es precisamente aquí donde interviene la tarea de selección vista anteriormente. Para Cosmides \& Tooby (1992), en algunas versiones de los experimentos en las que los sujetos se enfrentan con tareas de selección interviene un módulo mental diseñado para detectar a los tramposos en situaciones de intercambio social. Obviamente, este módulo fue producto de la selección natural y en ese sentido, según los psicólogos evolucionistas, funciona de manera adecuada cuando realiza tareas de selección en las que se involucra. Anteriormente se expuso una versión de una tarea de selección abstracta en la que la ejecución de los sujetos es muy pobre. Sin embargo, hay versiones de la tarea de selección en las que los sujetos mejoran su ejecución de manera importante. El siguiente es un ejemplo de Griggs \& Cox (1982):

Como una medida severa en contra de los conductores en estado de ebriedad, los oficiales de Massachussets están revocando licencias para el expendio de bebidas alcohólicas. Eres un portero de un bar de Boston, y perderás tu trabajo a menos que refuerces el cumplimiento de la siguiente ley: "si una persona toma cerveza, entonces debe tener más de 20 años de edad".

Las tarjetas de abajo muestran información acerca de cuatro personas sentadas en una mesa del bar donde laboras. Cada tarjeta representa solo a una persona. De un lado de la tarjeta aparece lo que la persona está tomando y del otro lado aparece la edad de la persona. Indica qué tarjetas tienes que necesariamente voltear para ver si alguna de estas personas está violando la ley.

\begin{tabular}{|c|c|c|c|}
\hline $\begin{array}{c}\text { Tomando } \\
\text { cerveza }\end{array}$ & $\begin{array}{c}\text { Tomando } \\
\text { refresco }\end{array}$ & 25 años & 16 años \\
\hline
\end{tabular}

\footnotetext{
${ }^{11}$ El trabajo de Mithen (1998), que en muchos aspectos es compatible con la psicología evolucionista, sostiene que incluso la mente del chimpancé así como la del eslabón perdido contaban ya con una inteligencia social especializada. Según Mithen, la inteligencia social fue la primera facultad modular que surgió en nuestros antepasados.

${ }^{12}$ Otro módulo que se encuentra íntimamente ligado a la cognición social es un módulo de la teoría de la mente, esto es, la capacidad de inferir estados mentales de otros sujetos. Según los psicólogos evolucionistas, frente a una tarea de razonamiento como la tarea de selección de tarjetas se involucra también la teoría de la mente para indicar cuáles serían los deseos y necesidades del otro, lo que permite entender la idea de costo-beneficio que supone la detección de tramposos (Ermer et al. 2006).

${ }^{13} \mathrm{Al}$ igual que otros módulos, para los psicólogos evolucionistas, el módulo de detección de tramposos es universal. Los niños a los 3 años entienden qué significa hacer trampa en una regla social, pero no qué significa violar una regla condicional que describa el mundo (Ermer, Cosmides \& Tooby 2007).
} 
Desde un punto de vista lógico, este problema es estructuralmente idéntico al problema expuesto anteriormente, pero su ejecución produce resultados significativamente distintos. Alrededor del 75\% de estudiantes universitarios obtuvieron una respuesta correcta a diferencia del $25 \%$ de estudiantes universitarios que tienen una respuesta correcta en la versión de la tarea de selección revisada anteriormente (Cosmides \& Tooby 1992).${ }^{14}$ Los psicólogos evolucionistas sostienen que experimentos como este apoyan la existencia de un módulo de detección de tramposos. ${ }^{15}$

Según Cosmides \& Tooby (1996), el resultado anterior, junto con otros descubrimientos, muestra que el razonamiento humano no está subsumido en mecanismos no normativos sino que es llevado a cabo por mecanismos modulares producto de la selección natural. De este y otros experimentos, Cosmides \& Tooby (1996), junto con otros psicólogos, han explícitamente sostenido que no es necesario preocuparnos por la interpretación pesimista de la racionalidad humana.

\section{Implicaciones en el debate acerca de la racionalidad}

La validez de las tesis y principios defendidos por los psicólogos evolucionistas ha sido debatida por distintos teóricos, los cuales han generado un impresionante número de investigaciones. Considero que en el campo filosófico es importante examinar cuáles serían las implicaciones acerca de la racionalidad humana, si la tesis de la modularidad masiva y la hipótesis de detección del tramposo fuesen correctas. En lo que resta del trabajo intentaré mostrar tales implicaciones y buscaré responder a la pregunta irealmente muestra la psicología evolucionista que la interpretación pesimista de la racionalidad humana está equivocada?

Comúnmente se asocia a la psicología evolucionista una visión de racionalidad optimista o, como Stanovich \& West (2003) la denominan, "panglosiana". Como he hecho anteriormente distinguiré, de momento, dos versiones de la in-

${ }^{14}$ Como parte de su explicación los psicólogos evolucionistas han intentado mostrar cómo este resultado apoya su propuesta y no otras opciones. Por ejemplo, se ha discutido si la buena ejecución en el caso del "portero en un bar" puede ser explicado por la familiaridad que la tarea representa. Para eliminar esta posibilidad Cosmides \& Tooby (1992) han diseñado experimentos que evocan problemas de la vida cotidiana en los cuales, según sus resultados, los seres humanos cometen errores de razonamiento. Al mismo tiempo, estos psicólogos presentan tareas que no son familiares a los sujetos, pero que involucran un contrato social en el que se debe detectar tramposos, y en el que los resultados son los adecuados. En otras palabras, para Cosmides \& Tooby (1992), la familiaridad de la tarea no explica la mejor ejecución en tareas como el "portero en un bar" y la mala ejecución en las versiones abstractas de la tarea de selección. Varios trabajos han puesto en duda la evidencia usada por Cosmides \& Tooby para apoyar su teoría, por ejemplo, Sperber \& Girotto (2003). En este trabajo, como he mencionado anteriormente, no discutiré la validez misma de los experimentos ofrecidos por los psicólogos evolucionistas o si el contenido en las tareas de razonamiento puede mejorar su ejecución o no, sino que me centraré en las implicaciones teóricas que sus estudios, de ser adecuados, tendrían en la manera en que se concibe la racionalidad humana.

${ }^{15}$ Hay dos módulos relacionados con el razonamiento humano que no abordaré en este trabajo: uno relacionado con el razonamiento acerca de riesgos (Ermer et al. 2006) y otro con el razonamiento frecuentista (Cosmides \& Tooby 1996). El primero de estos módulos, vinculado con el módulo de detección de tramposos, es un módulo que sirve para resolver reglas de precaución del tipo "si el sujeto S está involucrado en una actividad peligrosa, entonces S debe tomar precauciones". El otro módulo sirve para el razonamiento inductivo cuando este se presenta en formato de frecuencia. 
terpretación optimista de la racionalidad que podrían surgir de los trabajos hechos en la psicología evolucionista:

(A) Muchos fenómenos en los que los juicios de los sujetos concuerdan con las normas de racionalidad se explican por el hecho de que el razonamiento humano puede fundarse en módulos darwinianos que satisfacen las normas de la VER.

(B) Muchos fenómenos en los que los juicios de los sujetos concuerdan con las normas de racionalidad se explican por el hecho de que todo el razonamiento humano es llevado a cabo por módulos darwinianos que satisfacen las normas de la VER.

Las dos versiones de la interpretación optimista tienen en común hacer referencia a los mecanismos que subyacen el razonamiento humano, pero tienen un énfasis diferente en el número de mecanismos que satisfacen los principios de la VER. Mientras que en la primera versión la satisfacción de los principios normativos por parte de los sujetos se explica porque el razonamiento puede depender de mecanismos modulares que hacen un buen trabajo, en la segunda versión se afirma que el razonamiento está compuesto únicamente por mecanismos modulares normativamente no problemáticos, por lo que está garantizada la satisfacción de los principios normativos de razonamiento por parte de los sujetos. Así, según (A), si las tareas de razonamiento pueden ser llevadas a cabo por mecanismos modulares darwinianos, su resultado no se desviará de los principios normativos adecuados; no obstante, la versión (B), que resulta más fuerte, señala que estaría infundada cualquier preocupación acerca de la sistemática irracionalidad de los seres humanos, porque todos los mecanismos de razonamiento dependen de módulos darwinianos que satisfacen las normas de la VER.

Teniendo de telón de fondo las distintas versiones de la interpretación optimista de la psicología evolucionista es pertinente preguntarse si realmente la psicología evolucionista, con cada una de sus hipótesis, contrarresta la tesis pesimista de la racionalidad humana expuesta en la primera sección de este trabajo. Anteriormente distinguí dos versiones de la interpretación pesimista:

(I) Muchos fenómenos en los que los juicios de razonamiento se desvían de las normas de racionalidad se explican por el hecho de que el razonamiento humano puede fundarse en mecanismos que comúnmente no satisfacen las normas de la VER.

(II) Muchos fenómenos en los que los juicios de razonamiento se desvían de las normas de racionalidad se explican por el hecho de que los únicos mecanismos de razonamiento que los seres humanos poseen no satisfacen las normas de la VER.

Si se acepta la versión (A) de la interpretación optimista de la racionalidad humana, esto es, que muchos fenómenos en los que los juicios de los sujetos concuerdan con las normas de racionalidad se explican porque el razonamiento puede fundarse en módulos darwinianos, entonces se debe rechazar la versión pesimista (II). Presuponiendo que la hipótesis de la detección del tramposo fue- 
se correcta habría, al menos, un mecanismo de razonamiento que no es problemático. Sin embargo, según he expuesto, la psicología evolucionista no muestra que los seres humanos nunca cometan errores de razonamiento. ${ }^{16}$ Aun cuando la psicología evolucionista esté en lo correcto al mostrar que existen algunos mecanismos modulares que dan cuenta de competencias de razonamiento no problemáticas, no se sigue que los seres humanos no cometan errores fundados en mecanismos normativamente problemáticos. En otras palabras, aceptar la versión optimista (A) no supone el rechazo de la versión pesimista (I), es decir, (A) y (I) pueden ser compatibles. ${ }^{17} \mathrm{~A}$ este respecto, se podría sostener que mientras que la versión optimista (A) explica por qué los seres humanos pueden responder adecuadamente ciertos problemas de razonamiento, la interpretación pesimista (I) explica por qué los seres humanos no responden adecuadamente ciertos problemas de razonamiento.

La aceptación de la versión más optimista de la psicología evolucionista, esto es, la versión (B), que sostiene que todo el razonamiento humano está subsumido en módulos darwinianos que satisfacen las normas de la VER, supondría el rechazo de las versiones pesimistas (I) y (II) de la racionalidad humana. Aceptar que la cognición humana se funda en mecanismos normativamente no problemáticos supondría la falsedad de que todas o solo algunas de sus competencias de razonamiento violan los principios normativos -es decir, las versiones pesimistas (II) y (I), respectivamente-. De este modo, si se aceptara (B), entonces se tendrían contrarrestadas de manera completa las versiones de la interpretación pesimista (I) y (II) de la racionalidad humana.

Pero itenemos buenas razones para aceptar la versión optimista (B) o solo podemos aceptar la versión optimista (A)? Es posible argumentar que aun suponiendo que la mente cuenta con un módulo para detectar tramposos, de ahí no se podría sostener la versión optimista (B). El módulo de detección de tramposos, cuando es usado para resolver la tarea de selección de tarjetas que involucra la posible violación de un contrato social, supondría la existencia de solo un mecanismo que satisface los principios normativos adecuados, pero no que el ser humano solo posea mecanismos modulares ni tampoco que dichos mecanismos modulares sean todos normativamente adecuados. ${ }^{18} \mathrm{El}$ gran número de críticas a la teoría de la modularidad masiva también pondría en duda la idea de que los procesos mentales son llevados a cabo únicamente por módulos darwinianos (Fodor 2000, Buller 2005). Con ello, si bien la versión optimista (B) supondría la falsedad de las dos distintas versiones de la interpretación pesimista de la ra-

\footnotetext{
${ }^{16}$ Por ejemplo, en la tarea de selección de tarjetas en la versión original de Wason los sujetos cometen errores porque no se activa el módulo de detección de tramposos. Más adelante abordaré este tipo de cuestiones.

${ }^{17}$ Quiero dejar en claro que lo que sostengo aquí es que la versión optimista (A), que puede inferirse de la psicología evolucionista, puede ser compatible con la versión pesimista (I), que puede desprenderse de los resultados de la tradición de heurística y sesgo, y no que las teorías descriptivas defendidas por estas dos corrientes psicológicas sean en sí mismas compatibles.

${ }^{18}$ Se podría argumentar que el módulo de detección de tramposos no es el único que los psicólogos evolucionistas aceptan. Véase nota 15 . Sin embargo, considero que, aun aceptando la existencia de los módulos que ellos postulan en relación con el razonamiento, no se sigue la versión optimista (B).
} 
cionalidad humana, incluso suponiendo la validez del módulo de detección de tramposos y la existencia de otros módulos, de ahí no se podría justificar la verdad de la versión (B) de la interpretación optimista.

El análisis anterior parecería indicar que solo podemos aceptar la versión optimista (A). Sin embargo, los psicólogos evolucionistas no demuestran que la mera posesión de mecanismos modulares implique la satisfacción de la VER. Considero que habría tres posibilidades para dudar de ello:

i) Que la mente posea un módulo que no es normativamente problemático pero que no se activa, porque no tiene los inputs o insumos adecuados. Por ejemplo, la tarea de selección de tarjetas en la versión de Wason no activa el módulo de detección de tramposos. De este modo, aun cuando los sujetos cuenten con un módulo normativamente no problemático es necesario que dicho módulo se active para poder resolver apropiadamente una tarea de razonamiento.

ii) Que la mente posea mecanismos modulares que son activados por insumos que no eran propios al AAE y que por ese motivo ofrezcan respuestas que se desvían de un funcionamiento apropiado. Si bien una característica de los módulos es la especificidad de dominio, es posible que un módulo sea activado por un insumo que es parecido al que comúnmente lo activaba en el AAE, pero que al activarlo en un ambiente moderno conduzca a comportamientos no adaptativos. ${ }^{19}$ De este modo, no basta con tener módulos no problemáticos que simplemente se activen, sino que sean activados por los insumos adecuados.

iii) Relacionado con el punto anterior es posible que la mente posea mecanismos normativamente no problemáticos y que dichos mecanismos sean activados, pero que el ambiente ecológico en el que se desarrolla el ser humano moderno sea significativamente distinto al del AAE. ${ }^{20}$ Es fácil imaginar casos en los que una conducta en el pasado era adecuada para el $\mathrm{AAE}$, pero que resulta dañina en un ambiente diferente, como el actual. ${ }^{21}$

${ }^{19}$ Quizá la manera más simple de ilustrar este punto es que en el AAE de los ancestros humanos eran comunes las serpientes cuya mordedura pudo ser letal. Según los psicólogos evolucionistas, es posible que los seres humanos desarrollaran mecanismos psicológicos que rápidamente aprendieran a trasmitir temor o miedo, que a su vez desencadenará otro comportamiento, cuando se observaba una serpiente. A juicio de Cosmides y Tooby, este mecanismo fue diseñado para evitar un peligro particular, pero puede ser activado en la actualidad por algo parecido a una serpiente, como un juguete que tenga una forma similar. Los psicólogos evolucionistas constantemente señalan que es paradójico que los seres humanos modernos tengan miedo a las serpientes aun cuando representen un peligro sustancialmente menor que las tomas de luz en una sociedad occidental (Cosmides \& Tooby 1997). Sperber (1994) ha defendido una distinción entre dominio propio y dominio actual que en gran medida va en la misma dirección.

${ }^{20}$ El inciso iii), a diferencia del ii), señala que la producción de un comportamiento inadecuado se debe a que el ambiente actual se ha modificado aun cuando el insumo que originalmente activaba el módulo sea el mismo que en el AAE. El inciso ii), por su parte, señala que el comportamiento inadecuado se debe a que el módulo darwiniano es activado por insumos distintos al que originalmente lo activaba en el AAE.

${ }^{21}$ Algunas afirmaciones de los psicólogos evolucionistas apoyan estas ideas: "No todo comportamiento del que los organismos toman parte es adaptativo. El gusto por lo dulce puede ser adaptativo en ambientes en el cual las frutas ricas en vitaminas eran escasas, pero puede generar comportamientos poco adaptativos en ambientes modernos inundados de restaurantes de comida rápida" (Cosmides \& Tooby 1997). 
Considero que debido a i), ii) o iii) la simple posesión de módulos producto de la selección natural no basta para garantizar que los seres humanos cuentan con mecanismos normativos no problemáticos. ${ }^{22} \mathrm{Si}$ lo que aquí he argumentado es correcto, entonces la versión (A) de la interpretación optimista debe ser remplazada por:

(A') Muchos fenómenos en los que los juicios de los sujetos concuerdan con las normas de racionalidad se explican por el hecho de que el razonamiento humano puede fundarse en módulos darwinianos que satisfacen las normas de la VER, si (i) son activados, (ii) los insumos que los activan son los mismos o lo suficientemente similares a los que los activaban en el AAE y (iii) el ambiente actual es fundamentalmente similar al AAE. ${ }^{23}$

Hasta aquí, si se concediera la existencia de un módulo darwiniano de detección de tramposos y la idea misma de que la mente se compone de módulos, parece que únicamente habría motivos para aceptar (A'). Sin embargo, a mi juicio, hay todavía razones para pensar que el hecho de que la mente posea un módulo darwiniano que se active con los mismos insumos (o con insumos similares) a los que lo activaban en el AAE, dentro de un ambiente que es similar a AAE, de ahí no se sigue que siempre dichos módulos satisfagan las reglas provenientes de los principios de la lógica y la matemática, esto es, las normas de la VER.

Para aclarar este punto es necesario regresar a la idea de un mecanismo adaptativo dentro de la noción de módulo darwiniano. El hecho de que un módulo sea producto de la selección natural, como señalé anteriormente, supone que fue eficiente para resolver problemas adaptativos. Pero, a mi juicio, el que esos mecanismos hayan sido seleccionados para resolver esos problemas no implica que al resolverlos se sigan necesariamente las reglas de la lógica o la matemática. De hecho, muy probablemente esos mecanismos están en conformidad con las reglas de la lógica, pero nada nos muestra que su naturaleza sea tal que sigan las normas provenientes de la VER. Hay evidencia de que Cosmides \& Tooby aceptan esa idea.

\footnotetext{
${ }^{22}$ En términos generales, dado los resultados de los psicólogos evolucionistas, con ellos no pueden garantizar que un módulo que fue seleccionado para procesar cierto tipo de información en el AAE pueda ser normativamente adecuado para procesar otro tipo de información en un ambiente distinto a AAE. ¿Podría un módulo darwiniano procesar un insumo distinto al que procesaba en AAE y en un medio distinto a este ambiente y, no obstante, ser normativamente adecuado? Si esto fuera el caso, los psicólogos evolucionistas tendrían que sostener que esa función sería un subproducto de una función que se seleccionó para una función diferente. Los psicólogos evolucionistas conciben esta posibilidad cuando mencionan la posibilidad de subproductos: "Obviamente, somos capaces de resolver problemas que ningún cazador-recolector alguna vez ha tenido que resolver -podemos aprender matemáticas, conducir autos, usar las computadoras-. Nuestra capacidad para resolver otro tipo de problemas es un efecto secundario o subproducto de los circuitos que fueron diseñados para resolver problemas de adaptación" (Cosmides \& Tooby 1997, Principio 3).

${ }^{23}$ El agregado de (A') intenta recoger las razones i), ii) y iii) mencionadas anteriormente. Sin embargo, la manera en que he formulado este agregado puede ser cuestionable. Por ejemplo, se tiene el problema de cómo determinar que el ambiente en donde se desarrolla el ser humano actual es fundamentalmente similar al AAE. Dejaré estos problemas de lado para centrarme en otro aspecto que considero conduce al remplazo de la versión optimista $\left(A^{\prime}\right)$.
} 
Muchos mecanismos de dominio específico se activarán en un dominio y no en otro. Algunos de ellos incorporarán métodos racionales, pero otros llevarán a cabo procedimientos inferenciales que no responden a las formas lógicas sino [...] procedimientos que trabajan bien dentro de una estructura ecológica estable de un dominio particular, aun cuando podrían conducir a inferencias falsas o contradictorias si fuesen activadas fuera de tal domino (Cosmides \& Tooby 1997, Principio 4, el énfasis es mío).

Incluso haciendo aclaraciones en torno al módulo de detección de tramposos, los psicólogos evolucionistas afirman:

la definición de hacer trampa no se corresponde a la definición lógica de violación [...] las categorías lógicas y la definición de violación forman una categoría ortogonal (Fiddick, Cosmides \& Tooby 2000, p. 15, el énfasis es mío).

Si lo anterior es el caso, considero que se debe reformular $\left(A^{\prime}\right)$ por:

$\left(A^{*}\right)$ Muchos fenómenos en los que los juicios de los sujetos concuerdan con las normas de racionalidad se explican por el hecho de que el razonamiento humano puede fundarse en módulos darwinianos que son evolutivamente eficaces, ${ }^{24}$ si (i) son activados, (ii) los insumos que los activan son los mismos o lo suficientemente similares a los que los activaban en el AAE y (iii) el ambiente actual es fundamentalmente similar al AAE.

A mi juicio, suponiendo la existencia de un módulo de detección de tramposos e incluso la existencia de otros módulos darwinianos solo permiten a los psicólogos evolucionistas sostener, en relación con la racionalidad, la versión $\left(A^{*}\right)$. ¿Cuál es la relación de $\left(\mathrm{A}^{*}\right)$ con la interpretación pesimista de la racionalidad? En primer lugar, $\left(\mathrm{A}^{*}\right)$ es compatible con la versión pesimista (I), ${ }^{25}$ dando por supuesto el módulo de detección de tramposos, que este se active por los insumos adecuados y que el ambiente actual no sea distinto al AAE, demuestra que dicho módulo es biológicamente eficaz. Esta eficacia biológica a veces puede satisfacer los criterios de la VER; sin embargo, eso no niega que este módulo u otros mecanismos algunas veces no satisfagan dichos estándares.

De hecho, $\left(A^{*}\right)$ no solo es compatible con $(\mathrm{I})$, sino que podría explicar esta versión pesimista. Sostengo que con la versión optimista $\left(A^{*}\right)$ puede explicarse la versión (I) de la interpretación pesimista, porque dicha versión pesimista mantiene que muchos errores de razonamiento se deben a "mecanismos que comúnmente no satisfacen” las normas adecuadas. Una manera en que podría explicarse tal inadecuación es apelando a lo contemplado en la versión optimista $\left(A^{*}\right)$. Por ejemplo, en la versión optimista $\left(A^{*}\right)$ se afirma (en (ii)) que los mecanismos de razonamiento necesitan activarse por los mismos insumos o insu-

\footnotetext{
${ }^{24}$ Más adelante sugiero que los psicólogos evolucionistas están, en el fondo, planteando un criterio de racionalidad distinto al de la VER.

${ }^{25}$ Nuevamente, deseo subrayar que el hecho de que la versión optimista $\left(A^{*}\right)$ sea compatible con la versión pesimista (I) no significa que las teorías descriptivas de donde estas versiones surgen sean en sí mismas compatibles.
} 
mos suficientemente similares a los que los activaban en el AAE y, además, se pide (en (iii)) que el ambiente actual sea fundamentalmente similar al AAE; si se puede argumentar que estas dos condiciones no se cumplen (es decir, que las condiciones (ii) y (iii) no se cumplen), entonces los módulos darwinianos mismos se convertirían en mecanismos que "comúnmente no satisfacen" los estándares normativos adecuados. ${ }^{26}$ En otras palabras, la versión pesimista (I) podría ser explicada como casos en que no se cumplen las condiciones que $\left(\mathrm{A}^{*}\right)$ estipula o casos en que se cumple pero la eficacia evolutiva no corresponde con la satisfacción de los estándares provenientes de los principios de la lógica y la matemática. Si esto es correcto, entonces la propuesta supuestamente optimista defendida por los psicólogos evolucionistas podría conducir a una versión, ciertamente débil, de la interpretación pesimista, esto es, podría conducir a la versión pesimista (I).

¿Cuál es la relación de la versión optimista $\left(\mathrm{A}^{*}\right)$ con la versión pesimista (II)? A mi juicio, (A*) puede detener la fuerza de (II). Nuevamente, el módulo de detección de tramposos sería un caso en el que, si se activa con los insumos adecuados, como ocurre en las tareas de selección de tarjetas en las que se deben detectar tramposos, es eficiente y dicha eficiencia hace que algunas veces se cumpla con las normas de la VER.

Si lo que he argumentado hasta aquí es correcto, de aceptar la existencia del módulo de detección de tramposos y otros módulos defendidos por los psicólogos evolucionistas, nos quedamos con una postura de la racionalidad que a veces puede cumplir los estándares provenientes de la lógica y la matemática, como estipula la VER, pero que no fue diseñada para ello. Según la versión optimista $\left(\mathrm{A}^{*}\right)$, generalmente los juicios de los sujetos concuerdan con las normas de racionalidad por el hecho de que los seres humanos poseen módulos darwinianos que son evolutivamente eficaces, si (i) son activados, (ii) los insumos que los activan son los mismos o lo suficientemente similares a los que los activaban en el AAE y (iii) el ambiente actual es fundamentalmente similar al AAE. Pero, icuáles son las normas de racionalidad que los módulos darwinianos satisfacen? Sugiero que los psicólogos evolucionistas están proponiendo, de manera poco clara, un criterio distinto al usado por la VER para evaluar el razonamiento. En otras palabras, mi sugerencia es que los psicólogos evolucionistas están implícitamente aceptando una explicación normativa de la racionalidad diferente de la que asumen los psicólogos que defienden la interpretación pesimista de la racionalidad humana. ${ }^{27}$ Veamos.

\footnotetext{
${ }^{26}$ Si bien es posible inferir la versión pesimista (I) de la versión optimista $\left(A^{*}\right)$, no significa que dicha postura pueda ser explicada solo a través de dicha versión optimista. Los mecanismos que pueden dar lugar a sistemáticos errores de razonamiento de la versión pesimista (I) pueden ser producto de mecanismos que siguen las heurísticas propuestas por Kahneman y Tversky, a saber, la representatividad, la disponibilidad y el ajuste y anclaje (Kahneman, Slovic \& Tversky 1982).

${ }^{27}$ No hay muchas referencias que indiquen claramente cómo conciben la racionalidad los psicólogos evolucionistas. Sin embargo, hay afirmaciones que nos dan pistas de qué es lo que ellos tienen en mente. Por ejemplo, los psicólogos evolucionistas afirman "quizá sea tiempo de mostrar más respeto a nuestras intuiciones de lo que recientemente se ha hecho. Los mecanismos evolutivos que subyacen a nuestras intuiciones
} 
Como expuse en la sección II de este trabajo, los psicólogos evolucionistas afirman que la estructura cognitiva es producto de la selección natural. Bajo esta perspectiva, las mentes están compuestas de adaptaciones que tuvieron su origen en la selección natural durante la historia evolutiva de las especies. Las mentes, según Cosmides \& Tooby (1992), fueron "diseñadas" por la selección natural para resolver problemas adaptativos, esto es, la mente no está diseñada para resolver cualquier tipo de problema sino solo problemas adaptativos. Estos psicólogos consideran que los problemas adaptativos tienen dos características:

Primeramente, son problemas a los que la especie se enfrentó una y otra vez durante su historia evolutiva. En segundo lugar, son problemas cuya solución afectó la reproducción de un organismo individual -aun cuando la cadena pudo haber sido indirecta, o aun cuando el efecto en el número de descendencia pudo ser reducido [...]. La mayoría de los problemas adaptativos se relacionan con cómo los organismos conducen sus vidas: qué comen, quiénes se los comen, con quiénes se aparean, con quiénes socializan, cómo se comunican, y así sucesivamente. Los problemas adaptativos son el único tipo de problemas para los que la selección natural pudo diseñar circuitos para solucionarlos (Cosmides \& Tooby 1997).

Esto es, los mecanismos no están diseñados para resolver problemas como el de la selección de tarjetas en la versión de Wason (u otros problemas abstractos relacionados con el razonamiento deductivo o la probabilidad), sino problemas relacionados con la reproducción del individuo, donde es relevante la manera de socializar. En el caso del módulo de detección de tramposos los psicólogos evolucionistas afirman:

Los psicólogos interesados en el razonamiento lógico descubrieron que los sujetos tienen una ejecución pobre si se les solicita buscar violaciones de reglas condicionales en donde no se involucra la violación de contratos sociales. Pero la ejecución es excelente cuando las reglas condicionales involucran el intercambio social y la búsqueda de violaciones se corresponde a buscar tramposos. Pruebas posteriores mostraron que esto se debe no a que el intercambio social active las habilidades de razonamiento lógico, sino a que activa inferencias que son adaptativas cuando son aplicadas al intercambio social, pero no cuando son aplicadas a reglas condicionales que involucran otros temas (Ermer, Cosmides \& Tooby 2007, p. 139, el énfasis es mío).

Si lo anterior es el caso, un proceso cognitivo adecuado es aquel que es eficaz para resolver un problema adaptativo, y en tanto los problemas adaptativos son aquellos con los que el ser humano se enfrentó constantemente y que ayudan,

han estado a prueba por millones de años en un ambiente complejo y ricamente estructurado. Con solo algunos pocos cientos de años con las teorías normativas bajo nuestras espaldas, podría haber problemas del mundo real que seguramente se nos han escapado [...]. Pero cuando la intuición y la teoría de la probabilidad parecen chocar, parecería tanto lógico como prudente al menos considerar que podría haber una sofisticada lógica de la intuición. Podríamos descubrir que somos buenos estadistas intuitivos después de todo" (Cosmides \& Tooby 1996, p. 69, el énfasis es mío). A mi juicio, este tipo de afirmaciones (aunque en este contexto se habla solo del razonamiento inductivo) sugieren que los psicólogos evolucionistas aceptan que si las intuiciones fundadas en módulos chocan con los estándares de la VER (en este caso, la teoría de la probabilidad), entonces lo que se debe cambiar son los estándares de dicha visión de la racionalidad por una "lógica de la intuición" fundada en mecanismos evolutivos. 
directa o indirectamente, a la reproducción, entonces es posible sugerir que los psicólogos evolucionistas asumen un tipo particular de racionalidad, que denominaré "visión evolucionista de la racionalidad" (VEVOR), como sigue: un proceso cognitivo es racional si es eficaz para resolver problemas que están relacionados, directa o indirectamente, con la reproducción, y que se presentan constantemente a un individuo.

Lo primero que hay que notar es que la VEVOR se enmarca dentro de un acercamiento consecuencialista a la racionalidad, en tanto que evalúa a partir de las consecuencias a las que conduce un proceso cognitivo específico. El consecuencialismo particular en el que se inscribe la VEVOR es lo que se podría denominar consecuencialismo biológico, en el cual el razonamiento es evaluado a partir de su eficacia para alcanzar metas biológicas como la reproducción y la supervivencia. ${ }^{28}$ La VER, por su parte, se acerca más a una postura deontológica de la racionalidad en la que lo que es constitutivo del buen razonamiento es seguir las reglas apropiadas y no las consecuencias de seguir dichas reglas. Para la VER basta con satisfacer los principios de la lógica para considerar racional un proceso, independientemente de los efectos o consecuencias que se sigan de hacer uso de él.

No es claro que los psicólogos evolucionistas consideren que la VEVOR es el único estándar con el que se debe evaluar el razonamiento, pues el reto a la interpretación pesimista de la racionalidad humana parece sugerir que lo que ellos desean es negar esta evaluación pesimista haciendo uso del mismo estándar normativo asumido por la tradición de heurística y sesgo, es decir, la VER. No obstante, como he argumentado, el trabajo realizado por la psicología evolucionista puede alentar una manera de entender la interpretación pesimista de la racionalidad humana. Además, por el modo en que presentan sus avances y el modo en que conciben la cognición, he sugerido que los psicólogos evolucionistas se alejan de la VER para adoptar-quizá sin ser del todo conscientes de ellouna racionalidad evolucionista, i.e., la VEVOR. ${ }^{29}$

Haciendo uso de la distinción hecha entre tres distintos tipos de estudios de la razón, que apunté al inicio de este trabajo, es posible sostener que los psicólogos que defienden la interpretación pesimista de la racionalidad humana (en cualquiera de sus versiones) asumen la VER. Los psicólogos evolucionis-

\footnotetext{
${ }^{28}$ Esta manera de entender a la VEVOR es similar a la que Stanovich \& West (2003) denominan racionalidad evolutiva u optimización a nivel de los genes. Tanto la VEVOR como la optimización a nivel de los genes guardan similitud con la propuesta de racionalidad ecológica de Gigerenzer y su grupo. Un proceso es racional, según la propuesta de Gigerenzer, si da lugar a "tomas de decisiones que promueven las metas adaptativas de los organismos en un ambiente social o físico" (Gigerenzer \& Todd 1999, p. 364). Debido a que se aleja de los propósitos de este trabajo, no hago aquí una comparación de los distintos acercamientos a la racionalidad de corte evolutivo que han sido ofrecidos en la psicología cognitiva.

${ }^{29}$ La idea de que los psicólogos evolucionistas aceptan implícitamente la VEVOR, como he sugerido en esta sección, no es compartida por todos los teóricos. Samuels et al. (2004), por ejemplo, consideran que los psicólogos evolucionistas aceptan la VER. A mi juicio, hay elementos que apoyan la postura que defiendo en este trabajo, pero el hecho de que los psicólogos evolucionistas no sean explícitos con relación a la manera en que entienden los criterios usados para evaluar el razonamiento hace que esta cuestión no esté libre de controversias.
} 
tas que defienden explícitamente una versión optimista de la racionalidad parecen asumir un estándar normativo distinto, esto es, implícitamente aceptan que las normas apropiadas de razonamiento son caracterizadas por la VEVOR. Si la interpretación que hago de estos trabajos psicológicos es correcta, detrás del debate entre psicólogos pesimistas (como los defensores de la tradición de heurística y sesgo) y los psicólogos optimistas (como los psicólogos evolucionistas) parece haber una manera distinta de concebir las normas de razonamiento adecuadas. En otras palabras, detrás de un debate entre dos distintas teorías descriptivas del razonamiento (la tradición de heurística y sesgo y la psicología evolucionista) parece que se encuentra un debate entre dos distintas teorías normativas de la racionalidad.

\section{Conclusiones}

La psicología evolucionista considera que el razonamiento humano está fundado en módulos innatos producto de la selección natural. Un ejemplo particular de módulo, que he revisado en este trabajo, es aquel diseñado para lidiar con la detección de tramposos en contratos sociales. Con la postulación de este y otros módulos los psicólogos evolucionistas sostienen que están infundadas las preocupaciones en torno a la interpretación pesimista de la racionalidad humana. En este trabajo he distinguido dos versiones de la anterior interpretación y he puesto de manifiesto el estándar normativo que implícitamente presuponen. En particular, he argumentado que si la descripción del razonamiento humano que brinda la psicología evolucionista fuese correcta, esta apoyaría solo la versión optimista $\left(A^{*}\right)$, es decir, el razonamiento humano puede fundarse en módulos darwinianos que son evolutivamente eficaces, si i) son activados, ii) los insumos que los activan son los mismos o lo suficientemente similares a los que los activaban en el AAE y iii) el ambiente actual es fundamentalmente similar al AAE. Dando por supuestas las tesis de la psicología evolucionista, de ellas no se pueden sostener las versiones optimistas (A), (A') ni (B). Si (A*) es el caso, entonces es posible rechazar la versión pesimista (II) que sostiene que todos los mecanismos de razonamiento que los seres humanos poseen son normativamente problemáticos. Sin embargo, $\left(A^{*}\right)$ no ofrece razones para mostrar que los seres humanos no cometen errores "sistemáticos y severos" producto de mecanismos normativamente problemáticos, es decir, $\left(\mathrm{A}^{*}\right)$ resulta compatible con, e incluso podría explicar, la versión (I) de la interpretación pesimista de la racionalidad. Por último, he sugerido que la interpretación optimista $\left(\mathrm{A}^{*}\right)$ que defienden los psicólogos evolucionistas parece fundarse en una visión de la racionalidad distinta a la que asumen los defensores de la interpretación pesimista. Mientras que los psicólogos evolucionistas parecen implícitamente aceptar que el razonamiento debe ser evaluado a partir de la eficacia para alcanzar metas biológicas, como lo postula la VEVOR; los defensores de la tradición de heurística y sesgo sostienen que el razonamiento debe ser evaluado a partir de la satisfacción de las normas provenientes de la lógica y la matemática, como lo describe la VER. 


\section{Bibliografía}

Axelrod, R. (1996), La evolución de la cooperación, Madrid: Alianza Universidad.

Buller, D. (2005), Adapting Minds: Evolutionary Psychology and the Persistent Quest for Human Nature, Cambridge, MA: MIT Press.

Carruthers, P. (2006), The Architecture of Mind, Oxford: Oxford University Press.

Cherniak, C. (1986), Minimal Rationality, Cambridge, MA: MIT Press.

Cosmides, L. y J. Tooby (1992), "Cognitive adaptations for social exchange" en Barkow, J., Cosmides, L. y J. Tooby (eds.), The Adapted Mind. Evolutionary Psychology and the Generation of Culture, New York: Oxford University Press, pp. 163-228.

Cosmides, L. y J. Tooby (1994), "Origins of domain specificity: The evolution of functional organization", en Hirschfeld, L. y S. Gelman (eds.), Mapping the Mind: Domain specificity in cognition and culture, New York: Cambridge University Press, pp. 85-116.

Cosmides, L. y J. Tooby (1996), "Are human good intuitive statisticians after all? Rethinking some conclusions from the literature on Judgment under uncertainty", Cognition 58: $1-73$.

Cosmides, L. y J. Tooby (1997), "Evolutionary Psychology: A Primer”, http://www.psych. ucsb.edu/research/cep/primer.html Web (acceso 10 de diciembre 2010).

Elio, R. (2002), "Issues in common sense reasoning", en Elio, R. (ed.), Common sense, Reasoning, and Rationality, New York: Oxford University Press, pp. 3-36.

Ermer, E., Cosmides, L. y J. Tooby (2007), "Cheater-Detection Mechanism”, en Baumiester, R. F. y K. D. Vohs (eds.), Encyclopedia of Social Psychology, Thousand Oaks, CA: Sage Publications, pp. 138-140.

Ermer, E., Guerin, S., Cosmides, L., Tooby, J. y M. Miller (2006), "Theory of mind broad and narrow: Reasoning about social exchange engages ToM areas, precautionary reasoning does not", Social Neuroscience 1: 196-219.

Evans, J. (1991), "Theories of Human Reasoning: The fragmented state of the art", Theory and Psychology 1: 83-115.

Evans, J. y D. Over (1996), Rationality and Reasoning, East Sussex: Psychology Press.

Fiddick, L., Cosmides, L. y J. Tooby (2000), "No interpretation without representation”, Cognition 77: 1-79.

Fodor, J. (1983), The Modularity of Mind, Cambridge, MA: MIT Press.

Fodor, J. (2000), The Mind Doesn't Work that Way, The scope and limits of computational psychology, Cambridge, MA: MIT Press.

Gilovich, T., Griffin, D. y D. Kahneman (2002), Heuristics and biases: The Psychology of Intuitive Judgment, Cambridge: Cambridge University Press.

Goldman, A. (1993), Philosophical Applications of Cognitive Science, Boulder: Westview Press.

Gigerenzer, G. y P. M. Todd (1999), "What We Have Learned (So Far)", en Gigerenzer, G., Todd, P. M. y ABC Research Group (eds.), Simple heuristics that make us smart, New York: Oxford University Press, pp. 357-366.

Griggs, R. y J. Cox (1982), "The elusive thematic-materials effect in Wason's selection task", British Journal of Psychology, 73: 407-420. 
Kahneman, D., Slovic, P. y A. Tversky (1982), Judgment under uncertainty: Heuristics and biases, Cambridge: Cambridge University Press.

Mithen, S. (1998), Arqueología de la mente. Orígenes del arte, de la religión y la ciencia, Barcelona: Crítica.

Piatelli-Palmarini, M. (1994), Inevitable Illusions: How Mistakes of Reason Rule Our Minds, New York: John Wiley \& Sons.

Piatelli-Palmarini, M. (2005), Los túneles de la mente. ¿Qué se esconde tras nuestros errores?, Barcelona: Crítica.

Samuels, R., Stich, S. y L. Faucher (2004), "Reason and Rationality", en Niiniluoto, I., Sintonen, M. y J. Wolenski (eds.), Handbook of Epistemology, Dordrecht: Kluwer, pp. 131- 179.

Sperber, D. (1994), "The modularity of thought and the epidemiology of representations", en Hirschfeld, L. y S. Gelman (eds.), Mapping the Mind: Domain specificity in cognition and culture, New York: Cambridge University Press, pp. 39-67.

Sperber, D. y V. Girotto (2003), "Does the selection task detect cheater-detection?", en Sterelny, K. y J. Fitness (eds.), From Mating to Mentality. Evaluating Evolutionary Psychology, New York: Psychology Press, pp. 197-226.

Stanovich, K. y R. West (2003), "Evolutionary Versus Instrumental Goals: How Evolutionary Psychology Misconceives Human Rationality", en Over, D. (ed.), Evolution and the Psychology of Thinking: The debate, East Sussex: Psychological Press, pp. 171-221.

Stein, E. (1996), Without Good Reason. The Rationality Debate in Philosophy and Cognitive Science, Oxford: Claredon University Press.

Trivers, R. (1971), "The evolution of reciprocal altruism”, Quarterly Review of Biology 46: $35-56$.

Wason, P. (1966), "Reasoning”, en Foss, B. (ed.), New Horizons in Psychology, Harmondsworth: Penguin, pp. 135-151. 